\title{
Optimal Setting Method of Inverse Time Overcurrent Protection for the Distribution Networks Based on the Improved Grey Wolf Optimization
}

\author{
Bo Yang, ${ }^{1}$ Jun Tang, ${ }^{1}$ Changsen Feng ${ }^{D},{ }^{2}$ Chen Yang, ${ }^{1}$ and Xiaofeng Dong ${ }^{1}$ \\ ${ }^{1}$ State Grid Suzhou Power Supply Company, Suzhou 215004, China \\ ${ }^{2}$ College of Information Engineering, Zhejiang University of Technology, Hangzhou 310023, China
}

Correspondence should be addressed to Changsen Feng; fcs@zjut.edu.cn

Received 12 May 2021; Accepted 22 July 2021; Published 30 July 2021

Academic Editor: Xiaoqing Bai

Copyright ( $\odot 2021$ Bo Yang et al. This is an open access article distributed under the Creative Commons Attribution License, which permits unrestricted use, distribution, and reproduction in any medium, provided the original work is properly cited.

\begin{abstract}
The setting of protection parameters is vital to the large-scale application of reverse time overcurrent protection in the distribution networks. A fixed value optimization method of inverse time overcurrent protection for the distribution networks with distributed generation based on the improved Grey Wolf algorithm is proposed, which takes the protection action equation and the sensitivity, speed, and selectivity into consideration. Subsequently, four strategies, including good point set initialization, convergence factor exponential decay strategy, mutation strategy, and heuristic parameter determination strategy, are introduced to improve the Grey Wolf algorithm on the premise of retaining fewer adjustable parameters. Simulation results verify the feasibility and superiority of the proposed model in case of the two-phase and three-phase faults and discuss the influence of time differential on parameters setting and the research direction of algorithm optimization and engineering application.
\end{abstract}

\section{Introduction}

Distributed generation (DG), such as wind power, photovoltaic, fuel cell, and energy storage, is increasing access to the distribution networks along with China's nonignorable energy and environmental problems and the intelligent level of the power grid [1-4]. The widespread utilization of DG not only can ease the energy crisis and improve the utilization rate of energy but also will change the original grid structure and affect the sensitivity of protection. Therefore, the risk of refusing action or misoperation of the protection may be increased [5-7].

By far, research efforts mainly focused on two solutions:

(1) Collecting the electric parameters information of the critical nodes and processing them comprehensively to improve the protection of adjacent coordination characteristics and promoting the sensitivity of protection $[8,9]$ : according to the control strategy and fault current output characteristics of the inverter distributed power supply, an adaptive current velocity break protection scheme for the distribution networks is proposed in [8], and the power reference and control parameters can be obtained based on the MMS service of IEC61850 communication protocol. Literature [9] proposed a communication-based inverse time-limit overcurrent protection scheme, in which the upstream line protection receives the fault current data of the branch of the distributed generation by the communication channel to ensure the coordination between the upstream and the downstream protection. However, this method relies too much on the communication system, and if the communication system crashes, the protection will fail.

(2) The protection method suitable for large-scale DG distribution networks is improved to realize the adaptive protection setting. Inverse time overcurrent protection is widely applied in the distribution network protection scheme due to the characteristic of the short protection action time and stable 
operation [10-13]. Some researchers introduced the artificial intelligence algorithm to optimize the fixed value setting of the inverse time overcurrent protection. In [11], a fixed value optimization model of inverse time overcurrent protection based on an improved particle swarm algorithm is established, which considers the uncertainty of fault line, fault type, and fault point location. Nevertheless, the particle swarm optimization algorithm has too many adjustable parameters, and the parameters have a direct impact on the search quality and efficiency of the algorithm. In [12], two settings of fixed values are obtained by distinguishing two scenarios of twophase short circuit and three-phase short circuit, and the numerical example verifies that the sensitivity of a separate setting is more robust than that of a single setting. However, the method is restricted by too many parameters of particle swarm optimization (PSO). Literature [13] introduces the Grey Wolf algorithm to solve the fixed value setting of the inverse time overcurrent protection problem. The results show that the Grey Wolf algorithm has more advantages than the particle swarm algorithm in a high-dimensional nonlinear nonconvex optimization problem with inequality constraints. However, the Grey Wolf algorithm is easy to rapid convergence and needs to be improved.

Given the consideration above, a protection setting optimization method of inverse time overcurrent for distribution networks with DG based on the improved Grey Wolf algorithm is proposed. The good point set initialization strategy, convergence factor attenuation strategy, mutation strategy, and heuristic parameters strategy are introduced to develop the Grey Wolf algorithm, improving the algorithm accuracy without introducing a new parameter and retaining the advantages of the less adjustable parameter of the Grey Wolf algorithm. The simulation verified that the proposed model could effectively improve the selectivity and quick action of the inverse time overcurrent protection for the distribution networks with DG, guiding the corresponding scenario engineering practice.

\section{Setting Value Optimization of Inverse Time Overcurrent Protection for Distribution Networks}

2.1. Action Characteristic Equation. The inverse time overcurrent protection is widely used in the distribution network $[14,15]$. The characteristic equation of the inverse time overcurrent protection operation is as follows:

$$
t_{i j}=\frac{0.14 T_{p i}}{\left[\left(I_{i j} / I_{p i}\right)^{0.02}-1\right]},
$$

where $i$ is the number of the equipment, $j$ is the number of fault lines, $t_{i j}$ is the action time of the protection equipment, $T_{p i}$ is the time setting coefficient of the protection equipment, $I_{i j}$ represents the current flowing through the equipment, and $I_{p i}$ denotes the starting current of the equipment $i . T_{p i}$ and $I_{p i}$ are the value to be set.

2.2. Objective Function. Like other protection, the purpose of inverse time protection is to match the action time and short circuit current and timely remove the fault line in case of failure. In this study, the selectivity, reliability, and sensitivity are considered, and the matching of the action time and the short circuit current are guaranteed through the motion equation and constraint conditions. Given this premise, the least the total action time is, the better the protection sensitivity will be. Therefore, the protection setting can be formulated as a nonlinear optimization problem.

In [11-13], the objective function is minimizing the sum of all the action time of the relay. The target function of the setting value optimization of the inverse time overcurrent protection can be described as follows:

$$
O=\min \left[\sum_{i=1}^{M} \sum_{a=1}^{B} \sum_{j=1}^{L}\left(t_{i j}^{p}+t_{a j}^{b}\right)\right],
$$

where $M$ is the total number of the primary protection, $B$ is the number of backup protection, and $L$ represents the number of fault lines. $t_{i j}^{p}$ and $t_{a j}^{b}$ denote the action time of primary protection $i$ and backup protection $a$ in case of line failure, during which the relay must send a tripping signal.

2.3. Coordinate Constraint. Cooperation of primary protection and backup protection is crucial to the safe and stable operation of the power system. The time limit of protection should be determined step by step to guarantee the selectivity of protection operation at all levels. The primary protection and backup protection action time must have a reasonable interval to ensure the coordination and selectivity of relay protection.

Therefore, the protection coordination time interval should be satisfied:

$$
t_{i j}^{p}-t_{a j}^{b} \geq \mathrm{CTI}, \quad \forall i,\{j, k\},
$$

where CTI represents the coordinate interval for protection, also known as the time differential. Shortening the time differential can effectively increase the resection rate of the fault and reduce equipment damage.

2.4. Constraints. Firstly, the protection action time must meet the following constraints to ensure the sensitivity and quick action of the relay:

$$
t_{i j, \min } \leq t_{i j} \leq t_{i j, \max }
$$

where $t_{i j, \min }$ and $t_{i j, \max }$ are the minimum and maximum action time of the relay.

Secondly, the time setting coefficient and current setting coefficient must be within the scope of allowed values: 


$$
\begin{array}{r}
T_{p \min } \leq T_{p i} \leq T_{p \max }, \\
\forall i, \\
I_{p \min } \leq I_{p i} \leq I_{p \max }, \\
\forall i,
\end{array}
$$

where $T_{p \min }$ and $T_{p \max }$ are the maximum and minimum values of the time setting coefficient and $I_{p \min }$ and $I_{p \max }$ are the maximum and minimum starting current of the protection equipment.

To sum up, equations (1) and (2) are the objective function of the optimization model and equations (3)-(6) are the constraints.

\subsection{Influence of Large-Scale DG Access on Reverse Time} Overcurrent Protection in the Distribution Networks. As the large-scale DG access to the distribution networks, the bidirectional power appears. It needs to invest spare capacity and take control measures to compensate the intermittent power, which imposes a significant challenge on the protection setting and coordination operation of the distribution system. Moreover, the inverse time overcurrent protection may be affected by many aspects, such as the type of DG, access points, and distribution network mode.

2.5.1. The Impact of DG Type. Different kinds of DG, such as wind, solar, synchronous generator, fuel cell, and energy storage system, have different characteristics. Different types of DG regulate the active and reactive power in different ways. For example, wind power, usually asynchronous generators, needs to absorb reactive power from the grid without voltage regulation ability.

2.5.2. The Impact of DG Access Points. The short circuit current and the action time of the distribution network inverse time overcurrent protection depend on the access points and fault locations. DG access points have less impact on the upstream of the failure but affect the current downstream. If the downstream of the main protection action is faster, the upstream backup protection action time will be longer and may even refuse to move.

2.5.3. The Influence of DG Operation Mode. DG can parallel operation or run island, and there are many island schemes in DG island mode when switching. Different operation modes correspond to different grid topology structures and power flow, and the inverse time overcurrent protection may also be influenced.

The value setting is determined by solving optimization problems based on the distribution network topology structure containing DG access. It should be pointed out that the different topologies have covered most of the influencing factors. That is to say, no matter what type of the DG, what kind of DG operation mode from where the access points, and what the access scale, the setting optimization problem can be formulated by the similar mathematical optimization model, and this makes the optimization algorithm in this paper with general significance. In addition, when the capacity of DG access is over a specific value, the above optimization problem may not find the solution, which means that the inverse time overcurrent protection is not suitable for this scenario, and other suitable protection types should be introduced.

\section{Grey Wolf Algorithm}

Grey Wolf Optimizer (GWO), which has the advantages of stability and less regulating parameters, is a swarm intelligence algorithm presented by Mirijili in 2014 [16-18]. GWO can achieve an optimization search through the simulation of the Grey Wolf population hierarchy and hunting behavior. As shown in Figure 1, wolves can be divided into four types according to the status in the social structure of the Grey Wolf, including types $\alpha, \beta, \delta$, and $\omega$. Type $\alpha$ represents the group leader. Types $\beta$ and $\delta$ are in the middle level, and they assist type $\alpha$ and manage $\omega$. Type $\omega$ is at the bottom, and its function is to detect and prey. The four types are corresponding to the optimal solution, suboptimum solution, third optimal solution, and search populations of the intelligent algorithm, respectively.

Wolves find the optimal route of hunting by way of surrounding the prey, and the behavior can be modeled as

$$
\begin{aligned}
\vec{D} & =\left|\vec{C} \cdot \vec{X}_{p}(m)-\vec{X}(m)\right|, \\
\vec{X}(m+1) & =\vec{X}_{p}(m)-\vec{A} \cdot \vec{D},
\end{aligned}
$$

where $\vec{X}_{p}$ represents the current position of the prey, $\vec{X}$ denotes the location of the Grey Wolf, and $m$ is the number of iterations. $\vec{A}$ and $\vec{C}$ are the collaborative vector, and $\vec{D}$ is the distance between wolves and prey. The updated position of the Grey Wolf in the hunting process is formulated in equation (8).

Collaborative vectors $\vec{A}$ and $\vec{C}$ should be satisfied as follows:

$$
\begin{aligned}
& \vec{A}=2 \vec{a} \cdot \vec{r}_{1}-\vec{a}, \\
& \vec{C}=2 \cdot \vec{r}_{2},
\end{aligned}
$$

where the modulus values of $\vec{r}_{1}$ and $\vec{r}_{2}$ are a random number between $[0,1], \vec{a}$ is a convergence factor, and its value decreases linearly from two to zero with the increasing number of iterations.

The wolf hunting behavior is dominated by type $\alpha$, followed by type $\omega$. Type $\omega$ gradually moves to the highgrade wolf with ongoing hunting. Given these hunting behavior characteristics, the iterative optimization process of the Grey Wolf algorithm can be further described as follows: 


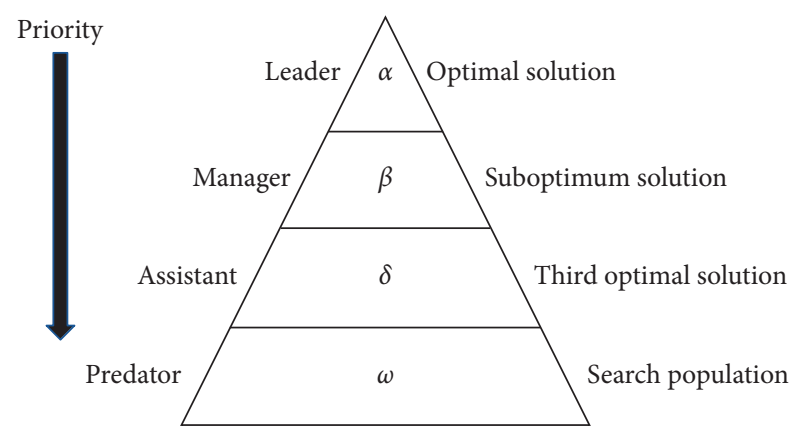

FIgURE 1: Hierarchy of GWO.

$$
\begin{gathered}
\left\{\begin{array}{l}
\vec{D}_{\alpha}=\left|\vec{C}_{1} \cdot \vec{X}_{\alpha}-\vec{X}\right|, \\
\vec{D}_{\beta}=\left|\vec{C}_{2} \cdot \vec{X}_{\beta}-\vec{X}\right|, \\
\vec{D}_{\delta}=\left|\vec{C}_{3} \cdot \vec{X}_{\delta}-\vec{X}\right|,
\end{array}\right. \\
\left\{\begin{array}{l}
\vec{X}_{1}=\vec{X}_{\alpha}-\vec{A}_{1} \cdot \vec{D}_{\alpha}, \\
\vec{X}_{2}=\vec{X}_{\beta}-\vec{A}_{2} \cdot \vec{D}_{\beta}, \\
\vec{X}_{3}=\vec{X}_{\delta}-\vec{A}_{3} \cdot \vec{D}_{\delta},
\end{array}\right. \\
\vec{X}(m+1)=\frac{\vec{X}_{1}+\vec{X}_{2}+\vec{X}_{3}}{3},
\end{gathered}
$$

where $\vec{X}_{\alpha}, \vec{X}_{\beta}$, and $\vec{X}_{\delta}$ represent wolf's position of types $\alpha$, $\beta$, and $\delta$ in the current iteration, respectively. $\vec{X}$ denotes the location of type $\omega . \vec{D}_{\alpha}, \vec{D}_{\beta}$, and $\vec{D}_{\delta}$ represent the distance between types $\alpha, \beta$, and $\delta$ with $\omega$ in the current iteration, respectively. $\vec{A}_{1}, \vec{A}_{2}, \vec{A}_{3}, \vec{C}_{1}, \vec{C}_{2}$, and $\vec{C}_{3}$ are the coordination coefficient and $\vec{X}(m+1)$ denotes wolf s location of type $\omega$ in the next iteration.

\section{Improved Grey Wolf Algorithm}

Like other intelligent algorithms, the Grey Wolf algorithm also faces prematurity and falling into local optimum $[19,20]$, limiting the accuracy of the algorithm, and has a large room for improvement. Therefore, the good point set initialization strategy, the convergence factor exponential decay strategy, the mutation strategy, and the heuristic parameter determination strategy are introduced to improve the Grey Wolf algorithm. While improving the accuracy of the algorithm, the improvement preserves the advantage of less adjustable parameters of the Grey Wolf algorithm.

4.1. Good Point Set Initialization Strategy. For the swarm intelligence optimization algorithm, the quality of the initial population greatly influences the global optimization effect and convergence speed. Generally, the initial population is generated based on random initialization, making it difficult to guarantee good population diversity. Therefore, the good point set strategy [21] is adopted to initialize the population distribution. Under the premise of taking the same number of points, the sequence initialized by the good point set is more uniform, and the generated initial population is more ergodic than others. It should be pointed out that it is conducive to the global optimization of the algorithm without increasing new algorithm parameters. The good point set was put forward by Hua Luogeng et al. It can be defined as follows.

Assume that $G_{s}$ is a unit cube of s-dimensional Euclidean space; the mathematical formulation can be described as follows:

$$
\begin{aligned}
P_{n}(k) & =\left\{\left(\left\{r_{1}^{(n)} * k\right\},\left\{\left\{r_{1}^{(n)} * k\right\}, \ldots,\left\{r_{s}^{(n)} * k\right\}\right), 1 \leq k \leq n\right\} r\right. \\
& \in G_{s} \varphi(n)=C(r, \varepsilon) n^{-1+\varepsilon},
\end{aligned}
$$

where $\varphi(n)$ is the deviation of $P_{n}(k)$ and $C(r, \varepsilon)$ is a constant that only depends on $\gamma$ and $\varepsilon$. $P_{n}(k)$ is called the good point set and $r$ is the good point.

4.2. Convergence Factor Exponential Decay Strategy. Convergence factor $\vec{a}$ affects the global search ability of the algorithm, and the exponential function is introduced to calculate $\vec{a}$. The convergence factor exponential decay strategy can replace the linear attenuation strategy of the basic GWO algorithm [22]:

$$
\vec{a}=2\left(1-\frac{m^{2}}{M^{2}}\right)
$$

where $m$ is the current iteration number and $M$ is the maximum iteration number. It can be seen from equation (13) that the convergence factor decreases nonlinearly with the increase of iterations, which helps to balance the global search ability and local optimization ability of the GWO algorithm.

4.3. Mutation Strategy. In order to solve the problems of prematurity and low convergence accuracy of GWO algorithm without introducing new parameters and increasing the complexity of parameter initialization, the Gaussian mutation strategy is adopted, and corresponding Gaussian mutation operator is given to the position iteration of Grey Wolf in equation (11), as shown in the following equation:

$$
\vec{X}(m+1)=\vec{X}(m+1) \cdot\left[1+\frac{|\vec{a}|}{2} \cdot N(0,1)\right],
$$

where $N(0,1)$ is the standard Gaussian distribution. This setting enables GWO to obtain enough perturbation at the initial stage of the algorithm to increase the global search ability of the algorithm and reduce the perturbation at the end of the algorithm to avoid the turbulence of the optimal solution and accelerate the convergence process.

4.4. Heuristic Parameter Determination Strategy. The parameters of the GWO algorithm (population size, maximum number of iterations) often have a direct impact on the 
search quality and efficiency of the algorithm. However, there is no simple, intuitive, and universal parameter determination method for the whole heuristic search strategy algorithm. In [23], the prior empirical setting method is utilized to directly give the parameters of the Grey Wolf algorithm and the comparison algorithm. Although the superiority of the target algorithm can be verified, the prior empirical setting method may be arbitrary when the target algorithm is applied to solve specific problems, and the comparison under different parameter settings is lacking. A heuristic parameter determination strategy is adopted to determine the parameters in two stages:

(1) According to the parameter range suggested in other literature, the population size is set to $10-50$, and the maximum number of iterations is set as 100-1000. Randomly select a set of parameters and perform optimization calculation.

(2) According to the influence of parameters on the GWO, the heuristic parameter determination strategy is adopted to adjust the initial parameters. The comparative analysis under different parameters is conducted, among which the parameters corresponding to the optimal result are the optimal parameters. The larger the population size and the more the maximum number of iterations, the greater the possibility of finding the optimal global solution will be, but the optimization time is longer.

One of the advantages of the improved GWO proposed in this paper is that no new parameters are introduced in the improvement process. The advantage of the Grey Wolf algorithm with fewer adjustable parameters is retained. Compared with the PSO algorithm and harmony search algorithm (HSA) with more parameters, the workload in the process of heuristic parameter determination is greatly reduced.

\section{Modeling}

In this study, equations (1) and (2) are taken as the objective function, and equations (3)-(6) are taken as the constraint conditions. Based on a specific distribution network topology structure, a setting value optimization model of inverse time-limit overcurrent protection for the distribution networks with DG based on the improved GWO is established. The structure of the improved GWO model is depicted in Figure 2, and the modeling process is as follows:

(1) Heuristic initialization of the population size and maximum iteration times.

(2) Initialize the population distribution by the good point set strategy.

(3) Calculate the fitness of individual Grey Wolf and save the information of wolf types $\alpha, \beta$, and $\delta$ with the best fitness.

(4) Update the location of Grey Wolf according to the convergence factor exponential attenuation strategy and mutation strategy.

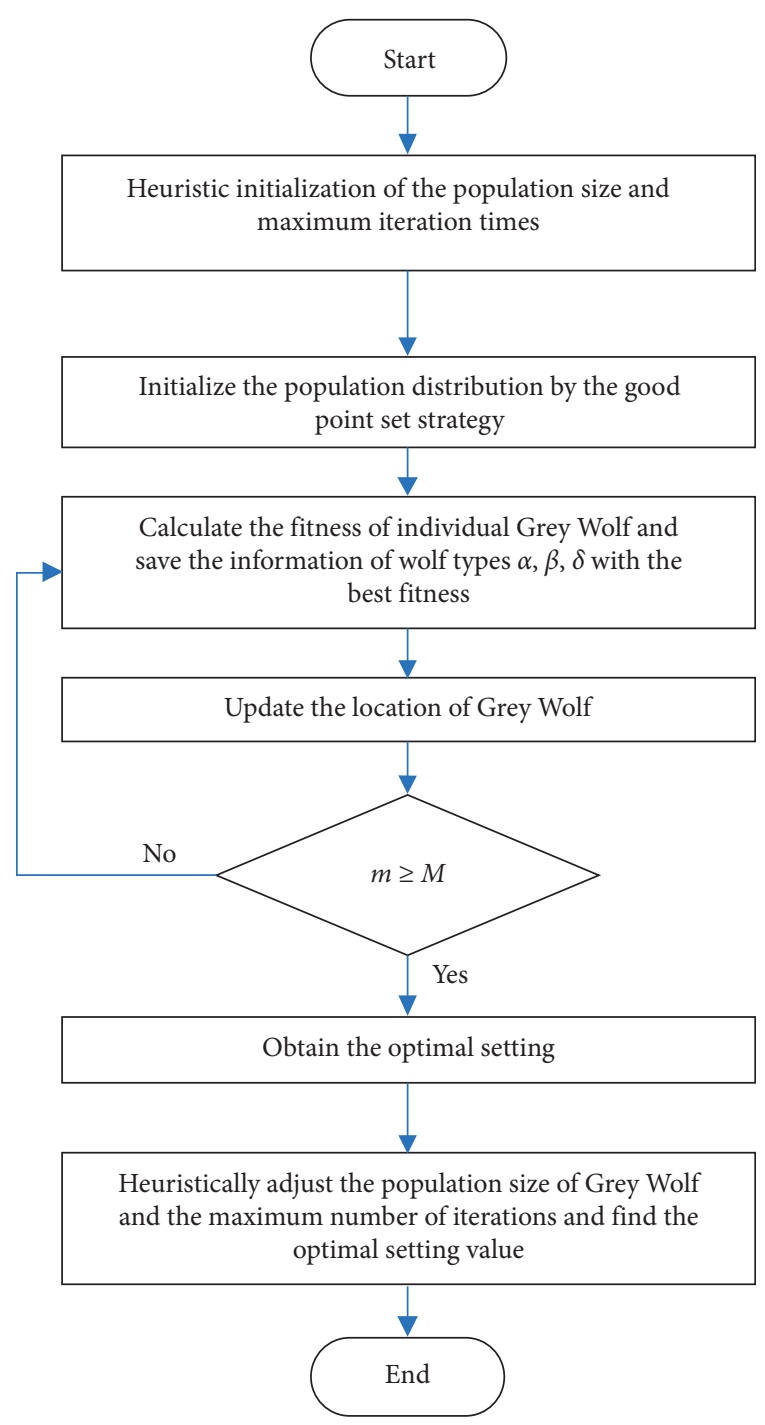

FIgURE 2: Improved GWO algorithm flowchart.

(5) If the maximum number of iterations is not reached, skip to Step 3 to continue optimization. Otherwise, the optimization ends, and the optimized setting is obtained.

(6) Heuristically adjust the population size of Grey Wolf and the maximum number of iterations and find the optimal setting value after several attempts and comparisons

\section{Case Study}

6.1. Example Description. In order to verify the effectiveness of the method presented in this paper, an example proposed in [12] is selected, and the improved GWO method is compared with the proposed setting method and other methods. The following IEEE 15-node distribution network with DG is employed to conduct the comparison analysis, as shown in Figure 3.

The parameter settings are listed in Table 1 , the maximum power of DG is $480 \mathrm{kVA}$, and the DG is at the 


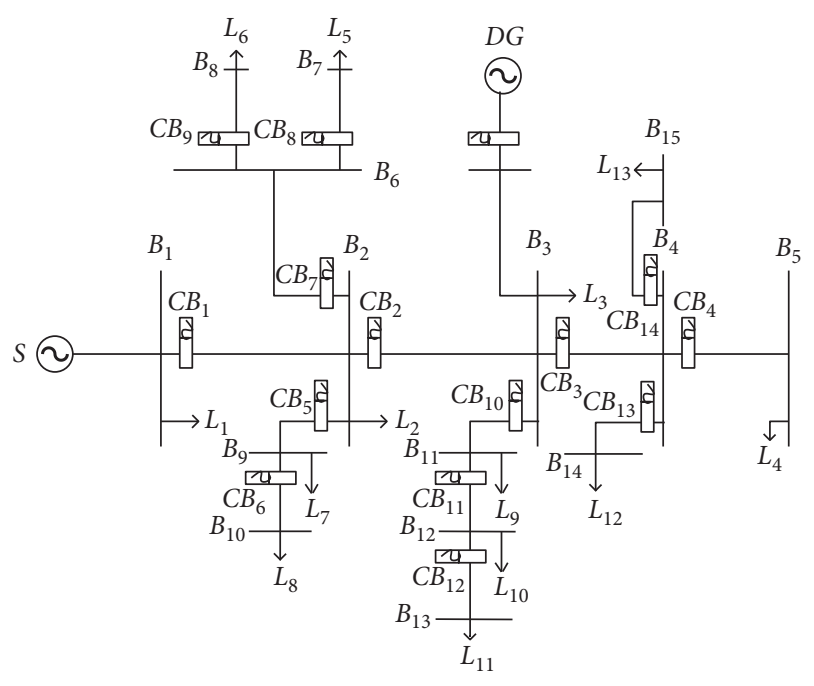

FIGURE 3: IEEE 15-bus distribution network with DG.

TABle 1: Parameter settings.

\begin{tabular}{lccc}
\hline Parameter & Value & Parameter & Value \\
\hline DG maximum power & $480 \mathrm{kVA}$ & $T_{p \min }$ & 0.1 \\
CTI & $0.5 \mathrm{~s}$ & $T_{p \max }$ & 0.4 \\
$t_{i j, \min }$ & $0.1 \mathrm{~s}$ & $I_{p \min }$ & $100 \mathrm{~A}$ \\
$t_{i j, \max }$ & $4 \mathrm{~s}$ & $I_{p \max }$ & $500 \mathrm{~A}$ \\
\hline
\end{tabular}

maximum output state. When CB1 is the primary protection, there is no backup protection. In other cases, there is one backup protection for each primary protection. The load parameters are shown in Table 2.

The improved PSO in [11], the improved HSA in [12], and the basic GWO and Cuckoo linear programming optimization algorithm (COA-LP) in [13] are selected to provide a comparative analysis. Two-phase short circuit and three-phase short circuit scenarios are distinguished in the optimization, and the protection value is set according to the terminal short circuit current. The system topology, protection method, objective function, and constraint conditions are all the same, and the quality of the optimization result mainly depends on the quality of the optimization algorithm. If the optimization results of the proposed method are superior to those of the control group, the effectiveness and superiority of the improved GWO algorithm in solving the problem of inverse time overcurrent protection setting can be verified.

6.2. Comparison Analysis. The results of the two-phase short circuit scenario are shown in Table 3. It can be seen from Table 3 that the total action time of the optimal solution obtained by the improved GWO algorithm is 23.162 seconds when a two-phase short circuit occurs, which is less than the other four algorithms.

In the case of the two-phase short circuit scenario, the setting values of each protection obtained by the improved GWO algorithm are listed in Table 4, and the action time results of each protection are shown in Table 5. As can be
TABLE 2: Load parameters.

\begin{tabular}{lcc}
\hline Load & Active power (MW) & Reactive power (MVar) \\
\hline 1 & 0.4 & 0.15 \\
2 & 0.1 & 0.2 \\
3 & 0.25 & 0.1 \\
4 & 0.3 & 0.2 \\
5 & 0.1 & 0.05 \\
6 & 0.3 & 0.07 \\
7 & 0.1 & 0.05 \\
8 & 0.05 & 0.02 \\
9 & 0.2 & 0.08 \\
10 & 0.1 & 0.1 \\
11 & 0.25 & 0.09 \\
12 & 0.1 & 0.05 \\
13 & 0.3 & 0.2 \\
\hline
\end{tabular}

TABLE 3: Results of five algorithms when a two-phase short circuit occurs.

\begin{tabular}{lc}
\hline Algorithms & Total action time (s) \\
\hline PSO & 30.812 \\
HSA & 24.573 \\
Basic GWO & 26.298 \\
COA-LP & 28.720 \\
Improved GWO & 23.162 \\
\hline
\end{tabular}

TABle 4: Protection setting value when a two-phase short circuit occurs.

\begin{tabular}{lcc}
\hline Relay & $T_{\mathrm{p}}$ & $I_{\mathrm{p}}(\mathrm{A})$ \\
\hline $\mathrm{CB}_{1}$ & 0.379 & 276.1 \\
$\mathrm{CB}_{2}$ & 0.301 & 193.2 \\
$\mathrm{CB}_{3}$ & 0.172 & 203.5 \\
$\mathrm{CB}_{4}$ & 0.099 & 104.7 \\
$\mathrm{CB}_{5}$ & 0.202 & 182.0 \\
$\mathrm{CB}_{6}$ & 0.108 & 106.9 \\
$\mathrm{CB}_{7}$ & 0.143 & 246.6 \\
$\mathrm{CB}_{8}$ & 0.101 & 113.7 \\
$\mathrm{CB}_{9}$ & 0.109 & 114.1 \\
$\mathrm{CB}_{10}$ & 0.203 & 232.5 \\
$\mathrm{CB}_{11}$ & 0.192 & 126.2 \\
$\mathrm{CB}_{12}$ & 0.105 & 103.9 \\
$\mathrm{CB}_{13}$ & 0.112 & 102.3 \\
$\mathrm{CB}_{14}$ & 0.097 & 101.9 \\
\hline
\end{tabular}

seen from Tables 3 and 5, the action time of protection obtained by the GWO algorithm satisfies the constraint of the time difference, which ensures selectivity and reliability of the protection and reduces the fault duration compared with other algorithms.

Similarly, the results of the three-phase short circuit scenario are shown in Table 6 . It can be found from Table 6 that the total action time of all protection obtained by the improved GWO algorithm in this paper is still the minimum in the case of a three-phase short circuit, which is better than the other four algorithms. The following conclusions can be drawn from the analysis:

(1) The good point set initialization strategy, convergence factor exponential decay strategy, mutation 
TABle 5: Protection action time when a two-phase short circuit occurs.

\begin{tabular}{lcc}
\hline Fault location & Action time of primary protection (s) & Action time of backup protection (s) \\
\hline $\mathrm{B}_{1}-\mathrm{B}_{2}$ & 1.119 & - \\
$\mathrm{B}_{2}-\mathrm{B}_{3}$ & 1.027 & 1.539 \\
$\mathrm{~B}_{3}-\mathrm{B}_{4}$ & 0.623 & 1.238 \\
$\mathrm{~B}_{4}-\mathrm{B}_{5}$ & 0.326 & 0.879 \\
$\mathrm{~B}_{2}-\mathrm{B}_{9}$ & 0.602 & 1.411 \\
$\mathrm{~B}_{9}-\mathrm{B}_{10}$ & 0.310 & 0.851 \\
$\mathrm{~B}_{2}-\mathrm{B}_{6}$ & 0.656 & 1.932 \\
$\mathrm{~B}_{6}-\mathrm{B}_{7}$ & 0.344 & 0.909 \\
$\mathrm{~B}_{6}-\mathrm{B}_{8}$ & 0.357 & 0.868 \\
$\mathrm{~B}_{3}-\mathrm{B}_{11}$ & 0.832 & 1.376 \\
$\mathrm{~B}_{11}-\mathrm{B}_{12}$ & 0.773 & 1.271 \\
$\mathrm{~B}_{12}-\mathrm{B}_{13}$ & 0.446 & 0.958 \\
$\mathrm{~B}_{4}-\mathrm{B}_{14}$ & 0.359 & 0.913 \\
$\mathrm{~B}_{4}-\mathrm{B}_{15}$ & 0.307 & 0.826 \\
\hline
\end{tabular}

TABLE 6: Results of five algorithms when a three-phase short circuit occurs.

\begin{tabular}{lc}
\hline Algorithms & Total action time $(\mathrm{s})$ \\
\hline PSO & 31.272 \\
HSA & 23.579 \\
Basic GWO & 25.195 \\
COA-LP & 28.209 \\
Improved GWO & 21.923 \\
\hline
\end{tabular}

strategy, and heuristic parameter determination strategy proposed in this paper can effectively improve the accuracy of the Grey Wolf algorithm

(2) No matter the two-phase short circuit or three-phase short circuit occurring, the improved GWO algorithm has a better optimization effect than the other four comparison algorithms

6.3. Heuristic Parameter Adjustment Analysis. The parameter settings of the optimization algorithm directly affect the search quality and efficiency of the algorithm. It should be pointed out that the Grey Wolf algorithm has only two adjustable parameters, which can save much work compared with PSO, HSA, and other methods in the parameter adjustment.

The population and the maximum number of iterations are adjusted based on the heuristic parameters optimization strategy. The number of wolves is set to 10,20,30, 40, and 50, respectively, and the maximum number of iterations of each group is set to 1000 . The optimizations are carried out to ensure the searching ability of the algorithm at the expense of time efficiency, and the optimal number of wolves can be determined. At the same time, the decreasing rate of the target value under the optimal number of wolves is observed to obtain the optimal iteration number.
The optimization results for the two-phase short circuit are shown in Table 7 . When the population size is 30 , the minimum value, average value, and standard deviation of the total operation time of protection are all the minimum, indicating that the population size of 30 is the most appropriate and has the best robustness.

The decline rate of the optimization target value is observed when the population size is 30 . It could be found that the algorithm converged when the average number of iterations reached 112 times, and therefore the maximum number of iterations is set as 150 .

Similarly, the population is set as 30 in the case of the threephase short circuit. The average number of iterations is 73 as the population converges, and the maximum number of iterations is set to 100. After the heuristic parameter adjustment, the algorithm parameters have better effectiveness and robustness, which can further improve the optimization quality.

6.4. Analysis of Time Differential. The CTI is simplified in this study, which is uniformly set as $0.5 \mathrm{~s}$. It should be noted that although $0.5 \mathrm{~s}$ is a typical time differential setting for protection, it is not optimal. Relay protection should speed up the operation time and shorten the time differential as far as possible under the condition of satisfying selectivity. Different time differentials can be selected for different protection coordination relationships. In engineering practice, it is necessary to set different time differential according to the actual system structure and protection type, combined with the experience of protection manufacturers and engineers.

The protection action results of the two-phase short circuit under different time differential are described in Table 8 . It can be seen from Table 8 that reducing the time differential can shorten the total operation time of the protection. Besides, the delay error depends on the type of protection device and the characteristics of the time relay. The settings should refer to engineering experience. 
TABLE 7: Results of heuristic parameter adjustment when a two-phase short circuit occurs.

\begin{tabular}{lcccc}
\hline Population size & $\begin{array}{c}\text { Maximum number of } \\
\text { iterations }\end{array}$ & Minimum total action time (s) & $\begin{array}{c}\text { Average total action time } \\
(\mathrm{s})\end{array}$ & $\begin{array}{c}\text { Standard deviation of the total } \\
\text { action time }\end{array}$ \\
\hline 10 & 1000 & 23.118 & 26.682 & 7.091 \\
20 & 1000 & 23.561 & 25.780 & 4.219 \\
30 & 1000 & 22.977 & 23.561 & 0.762 \\
40 & 1000 & 23.201 & 24.975 & 3.803 \\
50 & 1000 & 23.379 & 24.683 & 3.625 \\
\hline
\end{tabular}

TABLE 8: Protection action results of the two-phase short circuit under different time differential.

\begin{tabular}{lc}
\hline CTI $(\mathrm{s})$ & Total action time $(\mathrm{s})$ \\
\hline 0.4 & 22.381 \\
0.45 & 22.739 \\
0.5 & 23.162 \\
0.55 & 24.073 \\
0.6 & 24.833 \\
0.65 & 25.690 \\
0.7 & 27.196 \\
\hline
\end{tabular}

\section{Conclusions}

An optimization method based on the improved GWO is proposed to optimize the parameters setting of inverse time-limit overcurrent protection in the distribution networks with DG. According to the protection action characteristic equation and the sensitivity, speed, and selectivity of protection, the fixed value optimization model of inverse time-limit overcurrent protection for the distribution networks is established. The good point set initialization strategy, convergence factor attenuation strategy, mutation strategy, and heuristic parameters determination strategy are introduced to overcome the low convergence precision of the traditional GWO algorithm. Due to the few adjustable parameters, the workload in heuristic parameter determination is significantly reduced compared to the PSO algorithm and the HSA algorithm. Simulation results show that the proposed method has better effectiveness and robustness for both two-phase short circuits and three-phase short circuits and has good application value.

In the future, further research can be carried out from three directions: improving the action equation of inverse time limit protection, taking the time level difference as a part of the constraint conditions and optimization parameters, and exploring the better objective function.

\section{Data Availability}

All the data used in this study are presented in the manuscript. There are no extra data to be provided.

\section{Conflicts of Interest}

The authors declare that they have no conflicts of interest.

\section{Acknowledgments}

This work was supported by the World Class Urban Distribution Network Demonstration Project in Suzhou Historic District.

\section{References}

[1] C. Wei, Z. Shen, D. Xiao, L. Wang, X. Bai, and H. Chen, "An optimal scheduling strategy for peer-to-peer trading in interconnected microgrids based on RO and Nash bargaining," Applied Energy, vol. 275, 2021.

[2] A. M. Tsimtsios, G. N. Korres, and V. C. Nikolaidis, "A pilotbased distance protection scheme for meshed distribution systems with distributed generation," International Journal of Electrical Power \& Energy Systems, vol. 105, pp. 454-469, 2019.

[3] S.-E. Razavi, E. Rahimi, M. S. Javadi et al., "Impact of distributed generation on protection and voltage regulation of distribution systems: a review," Renewable and Sustainable Energy Reviews, vol. 105, pp. 157-167, 2019.

[4] S. Beheshtaein, R. Cuzner, M. Savaghebi, and J. M. Guerrero, "Review on microgrids protection," IET Generation, Transmission \& Distribution, vol. 13, no. 6, pp. 743-759, 2019.

[5] S. Mirsaeidi, D. M. Said, M. W. Mustafa, M. H. Habibuddin, and K. Ghaffari, "Fault location and isolation in micro-grids using a digital central protection unit," Renewable and Sustainable Energy Reviews, vol. 56, pp. 1-17, 2016.

[6] C. Wei, M. Benosman, and T. Kim, "Online parameter identification for state of power prediction of lithium-ion batteries in electric vehicles using extremum seeking," International Journal of Control, Automation and Systems, vol. 17, no. 11, pp. 2906-2916, 2019.

[7] M. N. Alam, "Adaptive protection coordination scheme using numerical directional overcurrent relays," IEEE Transactions on Industrial Informatics, vol. 15, no. 1, pp. 64-73, 2018.

[8] D. H. Zeng, G. Wang, J. M. Guo et al., "Adaptive current protection scheme for distribution network with inveterinterfaced distributed generators," Automation of Electric Power Systems, vol. 41, no. 12, pp. 86-92, 2017.

[9] Y. H. Guo, J. Jiang, C. J. Fan et al., "Improved inverse-time over-current protection for distribution network," Electric Power Automation Equipment, vol. 41, no. 12, pp. 86-92, 2017.

[10] V. R. Mahindara, D. F. C. Rodriguez, M. Pujiantara et al., "Practical challenges of inverse and definite-time overcurrent protection coordination in modern industrial and commercial power distribution system," IEEE Transactions on Industry Applications, vol. 57, no. 1, pp. 187-197, 2020.

[11] R. Guo, Research on Optimization of Inverse Time Overcurrent Protection Based on Improved Particle Swarm Optimization Algorithm, Yanshan University, Qinghuangdao, China, 2019. 
[12] K. J. Yang and C. Huang, "Optimal setting method of inverse time over-current protection for distribution network," Electric Power Automation Equipment, vol. 39, no. 3, pp. 163-168, 2019.

[13] J. Yi, Research on Optimal Coordination of Directional Overcurrent Relays in Microgrids Considering Different Network Topologies, Huazhong University of Science \& Technology, Wuhan, China, 2019.

[14] R. Jalilzadeh Hamidi, A. Ahmadian, R. Patil et al., "Optimal time-current graded coordination of multistage inverse-time overcurrent relays in distribution networks," International Transactions on Electrical Energy Systems, vol. 29, no. 5, Article ID e2841, 2019.

[15] E. C. Piesciorovsky and N. N. Schulz, "Fuse relay adaptive overcurrent protection scheme for microgrid with distributed generators," IET Generation, Transmission \& Distribution, vol. 11, no. 2, pp. 540-549, 2017.

[16] S. Mirjalili and A. Lewis, "Grey wolf optimizer," Advances in Engineering Software, vol. 69, no. 3, pp. 46-61, 2014.

[17] M. H. Nadimi-Shahraki, S. Taghian, and S. Mirjalili, "An improved grey wolf optimizer for solving engineering problems," Expert Systems with Applications, vol. 166, 2021.

[18] Q. Al-Tashi, S. J. Abdul Kadir, H. M. Rais, S. Mirjalili, and H. Alhussian, "Binary optimization using hybrid grey wolf optimization for feature selection," IEEE Access, vol. 7, pp. 39496-39508, 2019.

[19] H. Faris, I. Aljarah, M. A. Al-Betar, and S. Mirjalili, "Grey wolf optimizer: a review of recent variants and applications," Neural Computing and Applications, vol. 30, no. 2, pp. 413435, 2018.

[20] Z. Feng, D. Pei, and W. Wang, "Face recognition by support vector machine optimized by an improved grey wolf algorithm," Computer Engineering \& Science, vol. 41, no. 6, pp. 1057-1063, 2019.

[21] H. N. Zhang, X. M. You, S. Liu et al., "Ant colony algorithm based on dynamic evolution and interactive learning mechanism," Information and Control, vol. 49, no. 3, pp. 297-305, 2020.

[22] N. Mittal, U. Singh, and B. S. Sohi, "Modified grey wolf optimizer for global engineering optimization," Applied Computational Intelligence and Soft Computing, vol. 2016, no. 4598, 16 pages, Article ID 7950348, 2016.

[23] F. M. Tan, J. J. Zhao, and Q. Wang, "A grey wolf optimization algorithm with improved nonlinear convergence," Microelectronics \& Computer, vol. 36, no. 5, pp. 89-95, 2019. 